\title{
EXPOSIÇÃO DO CAVO DE MECKEL POR VIA EXTRADURAL NO TRATAMENTO DA NEURALGIA ESSENCIAL DO TRIGEMEO
}

\author{
Rolando A. Teruto*
}

Os excelentes resultados obtidos por Taarnhöj ${ }^{1}$, Love ${ }^{2}$ e Norlen ${ }^{3}$ com a descompressão das raízes e da porção posterior do gânglio de Gasser no tratamento da neuralgia essencial do trigêmeo, induziram-nos a tentar um método terapêutico ainda mais simples. Sem levar em conta as com. plicações graves, a anestesia e, mais particularmente, as parestesias que se instalam após a seç̧ão das vias sensitivas trigeminais justificam plenamente a procura de método cada vez menos mutilante para a neuralgia. do trigêmeo.

Cabe a Taarnhöj o mérito da concepção e divulgação de um processo que, sem interromper a continuidade das vias sensitivas da face, proporciona o desaparecimento das crises dolorosas da prosopalgia. Partindo da hipótese segundo a qual a neuralgia teria como causa a compressão da raiz posterior do trigêmeo, ao nível da porção mais alta da loja gas. seriana e, principalmente, no orifício através do qual esta raiz emerge na referida loja, Taarnhöj abre o cavo de Meckel por via intradural e secciona o anel fibroso por onde passa a raiz sensitiva do trigêmeo; nos 10 doentes assim operados o sucesso foi completo, desaparecendo as dôres sem o tributo dos efeitos colaterais conseqüentes às operações até então utilizadas.

Abrindo o cavo de Meckel em 3 casos de trigeminalgia mediante téc. nica algo modificada da original, isto é, expondo a raiz sensitiva próximo ao gânglio, utilizando via de acesso extradural, tivemos oportunidade de observar os mesmos brilhantes resultados referidos por Taarnhöj; em nenhum dos casos nos pareceu haver qualquer compressão da raiz retrogasseriana, a qual flutuava livremente no interior da sua loja fibrosa.

Êste fato levou-nos a tentar simplificar a operação e, sem abrir a loja gasseriana, completamente exposta por via extradural, observamos, em 4 casos, os mesmos resultados favoráveis proporcionados pela abertura do

Nota prévia apresentada no Departamento de Neuro-P'siquiatria da Associação Paulista de Medicina em 5 junho 1953.

* Chefe da Disciplina Neurocirúrgica do Departamento de Cirurgia do Hospital das Clínicas (Serviço dos Prof́s. Benedito Montenegro e Alípio Correia Neto). Chefe da Seção de Neurocirurgia da Enfermaria de Neurologia do Hospital das Clínicas (Serviço do Prof. A. Tolosa) da Fac. Med. da Ĺniv. de São Paulo. 
cavo de Meckel segundo a técnica de Taarnhöj ou pela variante por nós empregada inicialmente.

Sem pretender emitir qualquer opinião no tocante à patogenia da neuralgia essencial do trigêmeo, as verificações que fizemos quanto à não existência de compressão da raiz retrogasseriana dêste nervo, são, a nosso ver, suficientes para invalidar a hipótese patogênica formulada por Taarnhöj.

\section{SUMMARY}

\section{Exposure of the cavum Meckelli by extradural approach} in the treatment of major trigeminal neuralgia.

Report of a new technique for the treatment of major trigeminal neuralgia, consisting of exposure (with no opening) of the cavum Meckelli following an extradural approach. Four patients were operated on, with the same encouraging results obtained by others (Taarnhöj, Love, Norlen) employing different procedures. Although the author does not intend to give any explanation on pathogenesis of trigeminalgia he feels that the compression of the trigeminal roots inside the cavum Meckelli invoked by Taarnhöj as the mechanism of trigeminal neuralgia has lost its support.

\section{BIBLIOGRAFIA}

1. 'Taarnhöj, P. - Decompression of the trigeminal root and the posterior part of the ganglion as treatment in trigeminal neuralgia. Preliminary communication. J. Neurosurg., 9:288-290 (maio) 1952. 2. Love, G. J. - Decompression of the gasserian ganglion and its posterior root: a new treatment for trigeminal neuralgia (preliminary report). Proc. Staff Meet., Mayo Clin., 27:257-258 (jutho) 1952. 3. Norlen, G. - Cit. por Love 2. 